\title{
MICROENCAPSULAMENTO DO RESÍDUO DO PROCESSAMENTO AGROINDUSTRIAL DA UVA ISABEL (Vitis labrusca) POR GELIFICAÇÃO IONOTRÓPICA
}

\author{
A. C. G. VIERA ${ }^{1}$, G.C. FONTES ${ }^{2}$, M. H. M ROCHA-LEÃO ${ }^{2}$ e A. M. ROSSI ${ }^{3}$ \\ ${ }^{1}$ Universidade Federal do Rio de Janeiro , Instituto de Química \\ 2 Universidade Federal do Rio de Janeiro , Departamento de Engenharia Bioquímica \\ ${ }^{3}$ Centro Brasileiro de Pesquisas Físicas, Laboratório de Biomateriais \\ E-mail para contato: annacgv@gmail.com.br
}

\begin{abstract}
RESUMO - As indústrias que processam a uva no Brasil são na sua maioria vinícolas que consideram o bagaço (cascas e sementes) de uva como subproduto. Apesar de se tratar de um resíduo biodegradável, o acúmulo deste produto pode se tornar um sério problema ambiental. A importância em reutilizar o bagaço deve-se ao fato de seu conteúdo ser rico em compostos fenólicos, antioxidantes, antocianinas e corantes. A proposta deste trabalho foi otimizar o microencapsulamento do resíduo do processamento agroindustrial da uva isabel. As esferas foram preparadas por gotejamento da solução de alginato e do resíduo do processamento agroindustrial da uva isabel em solução de $\mathrm{CaCl}_{2}$. Para a otimização, foi realizado um planejamento fatorial fracionado $2^{4-1}$, variando as concentrações de alginato, resíduo do processamento agroindustrial da uva isabel, $\mathrm{CaCl}_{2}$ e tempo de reticulação. Os resultados obtidos neste trabalho indicam que o alginato é um biomaterial apropriado para o microencapsulamento do resíduo do processamento agroindustrial da uva isabel e com possibilidade de aplicação futura na indústria de alimentos.
\end{abstract}

\section{INTRODUÇÃO}

Nos últimos anos, a crescente preocupação dos consumidores com questões relacionadas à saúde tem alavancado o desenvolvimento de novos produtos com propriedades funcionais, que são aqueles que possuem compostos com potencial para retardar o estabelecimento de doenças e, com isso, melhorar a qualidade e a expectativa de vida ao promover a saúde (SGARBIERI,1999). Dentre os compostos com propriedades funcionais, um grande destaque tem sido dado aos antioxidantes, que ajudam a proteger o organismo humano contra o estresse oxidativo, associado a um aumento da incidência de câncer e outras doenças degenerativas (SCALBERT \& WILLIAMSON, 2000).

A uva é uma importante fonte de compostos fenólicos e antocianinas, no entanto, a quantidade e a composição destes compostos variam de acordo com a espécie, variedade, maturidade, tipo de cultivo e condições climáticas (Rizzon et al., 1998). 
A maioria das indústrias que processam a uva no Brasil são vinícolas que consideram o bagaço da uva como subproduto. Dados da indústria mostram que para 100 litros de vinho produzido geram-se $31,7 \mathrm{~kg}$ de resíduos, dos quais $20 \mathrm{~kg}$ são de bagaço (CAMPOS, 2005). Esses resíduos agroindustriais contêm uma variedade de espécies biologicamente ativas que são desperdiçadas, muitos deles ricos em compostos polifenólicos (CATANEO et al., 2008).

Apesar de se tratar de um resíduo biodegradável, o acúmulo deste pode acarretar um sério problema ambiental, uma vez que precisa de um tempo mínimo para ser mineralizado (CATANEO, 2008). Sendo de suma importância o estudo de formas de reaproveitamento pela indústria. Portanto, o objetivo do presente trabalho foi a produção de microesferas de alginato contendo a farinha da casca da uva (Vitis labrusca) para aplicação em espumante rose.

\section{MATERIAIS E MÉTODOS}

\subsection{Materiais}

- Alginato de sódio (Keltone® LV) - Apresenta uma proporção de ácido manurônico e ácido gulurônico $(\mathrm{M} / \mathrm{G})$ entre 0,4 e 1,9. Segundo o fabricante, uma solução de alginato de sódio 2 $\%(\mathrm{~m} / \mathrm{v})$ em água tem uma viscosidade a $25^{\circ} \mathrm{C}$ e $60 \mathrm{rpm}$ (spindle no. 2) de 100-300 mPa.s determinado pelo viscosímetro Brookfield modelo LV.;

- Cloreto de cálcio (Vetec®);

- As amostras de uva da variedade ISABEL (Vitis labruscas) foram adquiridas da Região do Vale do São Francisco, Estado de Pernambuco, cidade de Petrolina. Com o objetivo de preservar as propriedades gerais dos bagaços, os exemplares foram acondicionados em Ultrafreezer CL120-80V.

\subsection{Matéria seca}

O conteúdo de matéria seca dos bagaços da variedade de uva ISABEL foi desidratado em liofilizador Terroni Enterprise I, posteriormente cominuído em processador da marca Wallita e passados na peneira para análise (abertura em mm 0,5 e tyler 32) Granutest.

\subsection{Microencapsulamento do bagaço da uva}

As esferas contendo a casca da uva foram preparadas em matriz de alginato de sódio, pelo método de extrusão. Inicialmente, foram dissolvidos e homogeneizados, em becher de $25 \mathrm{~mL}$, separadamente, alginato de sódio $-0,25 \mathrm{~g} ; 0,35 \mathrm{~g} ; 0,45 \mathrm{~g}$; em $10 \mathrm{~mL}$ de água Milliq, e o extrato seco do bagaço da uva $(1 \% ; 2 \% ; 3 \%)$, através de movimentos manuais suaves para a prevenção de grumos e formação de bolhas nas soluções. Em seguida, as soluções recém preparadas foram misturadas e homogeneizadas para obtenção de uma suspensão formadora das esferas composta de alginato de sódio $(2,5 \% ; 3,5 \% ; 4,5 \%)$. 
Logo em seguida, gotejou-se a mesma, constantemente, em uma solução de cloreto de cálcio $\left(\mathrm{CaCl}_{2}\right)$, de concentração definida contida em erlenmeyer de $250 \mathrm{~mL}$. As concentrações molares das soluções de cloreto de cálcio foram de $0,15 \mathrm{M}, 0,2 \mathrm{M}$ e $0,45 \mathrm{M}$. Assim, foram formadas as cápsulas, que permaneceram por $15 ; 25$ ou 35 minutos na solução contendo os íons cálcio $\left(\mathrm{Ca}^{+2}\right)$, sendo coletadas por filtração e lavadas em seguida com água destilada. As esferas foram pesadas úmidas na balança analítica Ohaus Adventurer e com auxílio do paquímetro $\left(\right.$ Brasfort $^{\circledR}$ ) tiveram o seu tamanho determinado, posteriormente uma parte foi seca a $30^{\circ} \mathrm{C}$ em estufa de secagem Quimis, durante 24 horas e a outra parte foi seca no liofilizador.

\subsection{Otimização do grau de erosão e de intumescimento do encapsulado do pó da casca de uva nas esferas de alginato reticulado com cálcio}

Planejamento fatorial fracionado $2^{4-1}$ : Para otimizar o grau de erosão e de intumescimento do pó da casca da uva nas esferas, foi aplicado um delineamento fatorial, com o uso do programa de computação Statistica 7.0.

Inicialmente foi realizado um planejamento fatorial fracionado $2^{4-1}$, sendo os parâmetros fixados e adotados como variáveis independentes: concentração de alginato, casca da uva, cloreto de cálcio e tempo de reticulação. A Tabela 1 apresenta os limites para cada parâmetro estudado. As esferas foram preparadas como descrito no item 3.3.

A variável de resposta utilizada foi o grau de intumescimento e de erosão. Os experimentos foram realizados aleatoriamente.

Tabela 1. Fatores e níveis a serem analisados no planejamento experimental $2^{4-1}$

\begin{tabular}{cccc}
\hline & \multicolumn{3}{c}{ Nível $(\mathbf{g} / \mathbf{L})$} \\
\cline { 2 - 4 } Variáveis independentes & $\mathbf{- 1}$ & $\mathbf{0}$ & $\mathbf{+ 1}$ \\
\hline Alginato $(\% \mathbf{~ m} / \mathbf{v})$ & 2,5 & 3,5 & 4,5 \\
Casca da uva $(\% \mathbf{m} / \mathbf{v})$ & 1 & 2 & 3 \\
Concentração $\mathbf{C a C l}(\mathbf{M})$ & 0,15 & 0,2 & 0,45 \\
Tempo de reticulação $(\mathbf{m i n})$ & 15 & 25 & 35 \\
\hline
\end{tabular}

\subsection{Caracterização das esferas}

Estudo do grau de intumescimento das esferas: Os ensaios de intumescimento foram realizados para examinar a capacidade de hidratação de cada polímero ou sistemas de polímeros e avaliar o efeito complementar da influência que este fenômeno pode ter na cinética de liberação da casca da uva a partir das esferas de alginato. As esferas, após produzidas, eram lavadas com água Milli-Q, cujo excesso de água era retirado com papel de filtro para determinação de sua massa inicial. As esferas eram secas á $40^{\circ} \mathrm{C}$, até que a massa não variasse; logo após, a massa seca era anotada e a água de hidratação determinada pela equação abaixo. 


$$
\boldsymbol{G I}(\%)=\frac{\left(m_{u}-m s\right)}{m_{g}} \times 100
$$

Onde: $G I(\%)=$ Porcentagem de intumescimento das esferas, $\boldsymbol{m}_{\boldsymbol{u}}=$ massa úmida das esferas;

$\boldsymbol{m}_{\boldsymbol{s}}=$ massa das esferas secas;

Foram também realizados estudos de intumescimento das esferas secas ao longo do tempo após a imersão no espumante. Em intervalos de tempo pré-estabelecidos, retirou-se a água em excesso das esferas com o auxílio de um papel absorvente e realizou-se a pesagem, caracterizando a massa úmida $\left(\boldsymbol{m}_{u}\right)$. A análise foi realizada com quatro repetições.

Estudo do grau de erosão das esferas: Essa determinação foi baseada no trabalho de Efentakis et al (2000), com algumas modificações. As esferas secas foram armazenadas em tubo falcon contendo $20 \mathrm{~mL}$ de espumante. Após o intervalo de tempo selecionado, uma amostra de 4 esferas eram retiradas e ligeiramente secas em estufa para remoção da água e, em seguida, pesadas para registrar a diferença da massa comparada à amostra que não foi submetida ao teste. A perda de peso $(\mathrm{GE} \%)$ foi determinada seguindo a equação abaixo:

$$
G E(\%)=\frac{\left(P_{i}-P_{f}\right)}{P_{i}} \times 100
$$

Onde, GE, é a porcentagem do grau de erosão, $\boldsymbol{P}_{i}$ é o peso inicial da matriz seca e $\boldsymbol{P}_{f}$ é o peso da matriz após secagem em estufa, depois de determinado período de tempo no meio de dissolução.

Tempo de sedimentação: Os ensaios do tempo de sedimentação foram realizados para examinar o tempo que as microcápsulas levariam para percorrer do alto ao fundo do tubo de ensaio.

\section{RESULTADOS E DISCUSSÃO}

Os resultados obtidos na realização do planejamento fatorial fracionado $2^{4-1}$ são apresentados nas Tabelas 2 e 3, junto com as condições para cada um dos experimentos, assim como o grau de intumescimento e erosão das esferas. As tabelas 2 e 3 apresentam o grau de intumescimento e erosão das esferas liofilizadas e das secas na estufa respectivamente. Os dados indicam que o grau de erosão variou de 20,3 à 63,7 na tabela 2 e de 24,3 à 62,8 na tabela 3, com os diferentes níveis das variáveis independentes. O grau de intumescimento variou de 224,4 à 436,6 na tabela 2 e de 119,2 à 261,8 na tabela 3, também com os diferentes níveis das variáveis independentes.

Os dados apresentados nas Tabelas 2 e 3 não podem ser interpretados de forma direta, porque se faz necessária uma avaliação da significância dos parâmetros estudados por bases estatísticas. $\mathrm{Na}$ análise de variância pela ANOVA existe um parâmetro estatístico denominado $p$ - 
level, que permite avaliar quais fatores são estatisticamente relevantes. Quando os valores do $p$ level para cada um dos fatores e interações são menores ou iguais a 0,05 , estes apresentam significância ou relevância estatística, quando são maiores que 0,05 , os fatores e interações não apresentam relevância estatística (NETO et al., 1995; MONTGOMERY, 1999).

Tabela 2. Experimentos gerados pelo software Statistica 7.0 para o planejamento fracionado do encapsulado liofilizado.

\begin{tabular}{|c|c|c|c|c|c|c|}
\hline $\begin{array}{c}\mathbf{N}^{\mathbf{o}} \\
\text { Exper. }\end{array}$ & Alginato & $\begin{array}{l}\text { Casca } \\
\text { da } \\
\text { uva }\end{array}$ & $\begin{array}{c}\text { Concentração } \\
\text { de } \mathrm{CaCl}_{2}\end{array}$ & $\begin{array}{l}\text { Tempo de } \\
\text { reticulação }\end{array}$ & $\begin{array}{l}\text { Grau } \\
\text { de } \\
\text { erosão }\end{array}$ & $\begin{array}{c}\text { Grau de } \\
\text { intumescimento }\end{array}$ \\
\hline 1 & -1 & -1 & -1 & -1 & 20,3 & 306,8 \\
\hline 2 & +1 & -1 & -1 & +1 & 51,4 & 281,0 \\
\hline 3 & -1 & +1 & -1 & +1 & 36,6 & 396,3 \\
\hline 4 & +1 & +1 & -1 & -1 & 30,7 & 323,9 \\
\hline 5 & -1 & -1 & +1 & +1 & 63,7 & 436,6 \\
\hline 6 & +1 & -1 & +1 & -1 & 55,8 & 241,1 \\
\hline 7 & -1 & +1 & +1 & -1 & 62,1 & 350,0 \\
\hline 8 & +1 & +1 & +1 & +1 & 47,5 & 224,4 \\
\hline 9 & 0 & 0 & 0 & 0 & 41,4 & 328,1 \\
\hline 10 & 0 & 0 & 0 & 0 & 39,6 & 362,1 \\
\hline 11 & 0 & 0 & 0 & 0 & 44,2 & 344,8 \\
\hline
\end{tabular}

Tabela 3. Experimentos gerados pelo software Statistica 7.0 para o planejamento fracionado do encapsulado seco na estufa.

\begin{tabular}{ccccccc}
\hline $\begin{array}{c}\mathbf{N}^{\mathbf{0}} \\
\text { Exper. }\end{array}$ & Alginato & $\begin{array}{c}\text { Casca } \\
\text { da } \\
\text { uva }\end{array}$ & $\begin{array}{c}\text { Concentração } \\
\text { de } \mathbf{C a C l}_{2}\end{array}$ & $\begin{array}{c}\text { Tempo de } \\
\text { reticulação }\end{array}$ & $\begin{array}{c}\text { Grau } \\
\text { de } \\
\text { erosão }\end{array}$ & $\begin{array}{c}\text { Grau de } \\
\text { intumescimento }\end{array}$ \\
\hline $\mathbf{1}$ & -1 & -1 & -1 & -1 & 42,2 & 261,8 \\
\hline $\mathbf{2}$ & +1 & -1 & -1 & +1 & 24,3 & 171,2 \\
\hline $\mathbf{3}$ & -1 & +1 & -1 & +1 & 41,7 & 166,1 \\
\hline $\mathbf{4}$ & +1 & +1 & -1 & -1 & 29,0 & 147,8 \\
\hline $\mathbf{5}$ & -1 & -1 & +1 & +1 & 62,8 & 187,0 \\
\hline $\mathbf{6}$ & +1 & -1 & +1 & -1 & 56,6 & 142,3 \\
\hline $\mathbf{7}$ & -1 & +1 & +1 & -1 & 54,1 & 246,9 \\
\hline $\mathbf{8}$ & +1 & +1 & +1 & +1 & 49,5 & 158,4 \\
\hline $\mathbf{9}$ & 0 & 0 & 0 & 0 & 39,7 & 130,4 \\
\hline $\mathbf{1 0}$ & 0 & 0 & 0 & 0 & 47,3 & 155,9 \\
\hline $\mathbf{1 1}$ & 0 & 0 & 0 & 0 & 38,3 & 119,2 \\
\hline
\end{tabular}

A magnitude dos efeitos estimados de cada variável é apresentada nos diagramas de Pareto (Figuras 1,2,3 e 4), fornecendo o efeito quantitativo estimado que cada uma das variáveis 


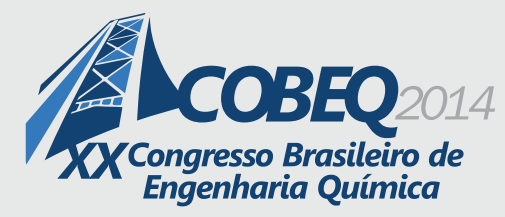

possuem sobre o grau de intumescimento e de erosão, estabelecendo quais desses efeitos encontram-se dentro do grau de confiança estabelecido para a análise $(95 \%)$.

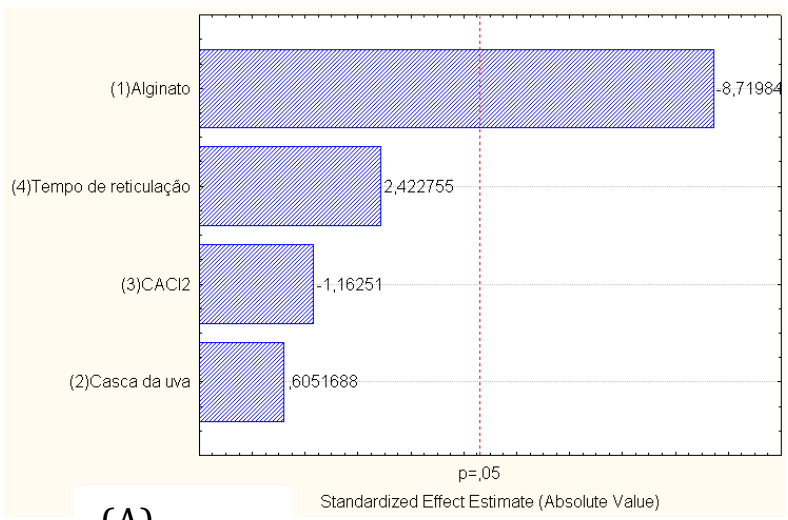

(A)

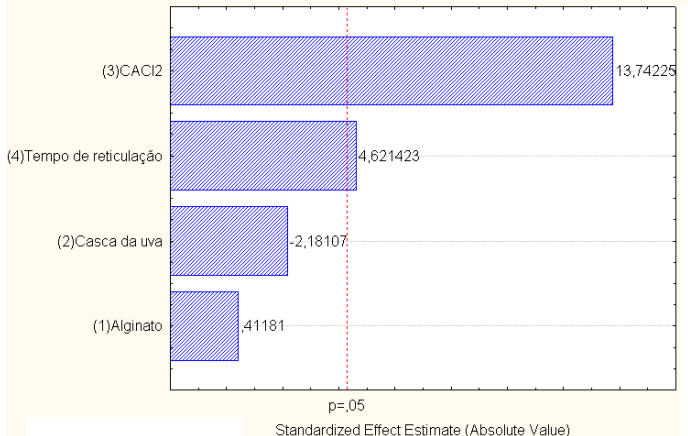

(B)

Figura 1. Diagrama de Pareto para o grau de intumescimento (A) e grau de erosão (B) do encapsulado liofilizado de cada variável do planejamento fatorial facionado $2^{4-1}$

Com os resultados da análise estatística das Figuras 1 (A) e 2 (A), confirmou-se que a variável com maior efeito sobre o grau de intumescimento foi a concentração de alginato. $\mathrm{O}$ efeito da concentração de alginato é negativo, indicando que níveis maiores de alginato levariam ao menor grau de intumescimento. Deve-se ao fato das esferas contendo maior teor de alginato serem capazes de ligar-se de forma mais eficiente aos íons cálcio, apresentando portanto menor intumescimento. Entretanto as variáveis concentração da casca da uva, o tempo de reticulação e a concentração de $\mathrm{CaCl}_{2}$ não foram estatisticamente significativos, ou seja, isso mostra que estes fatores na faixa estudada não influenciaram o grau de intumescimento.

A capacidade das esferas de alginato de intumescer é facilitada pelos grupos carboxílicos e hidroxilas, que se associam fortemente às moléculas de água. Um aumento no grau de reticulação diminui a disponibilidade desses grupos e, consequentemente, a hidrofilicidade do sistema. O grau de intumescimento é uma propriedade importante na predição do comportamento das cápsulas que serão utilizados em liberação controlada, pois modificações na estrutura da matriz polimérica causadas pelo intumescimento influenciarão na difusividade do antimicrobiano através do matriz (ZACTITI, 2006).

Esse resultado corrobora os estudos de Yu et al. (2010) que, ao avaliarem a variação da concentração de alginato no microencapsulamento de proteína do soro (BSA) como fármaco modelo em matriz de alginato, quitosana e pectina, observaram que, quanto maior a concentração de alginato, maior a eficiência de encapsulamento. Resultados similares também foram encontrados nos trabalhos de Anjani et al. (2007).

Na figura $2 \mathrm{~B}$ o resultado da análise estatística mostrou que a variável com maior efeito sobre o grau de erosão foi a concentração de $\mathrm{CaCl}_{2}$, seguido pelo tempo de reticulação, tendo 
ambas efeito positivo. Indicando que maiores níveis de $\mathrm{CaCl}_{2} \mathrm{e}$ de tempo de reticulação levariam a maiores porcentagens de grau de erosão. O mesmo efeito é encontrado no grau de erosão do encapsulado liofilizado (Figura 1 B). A concentração de cloreto de cálcio é de grande importância para a gelificação, processo que ocorre devido à afinidade entre o alginato e o cálcio.

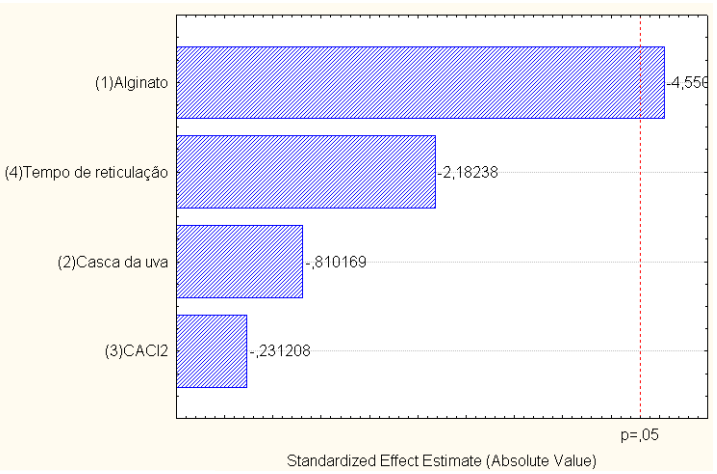

(A)

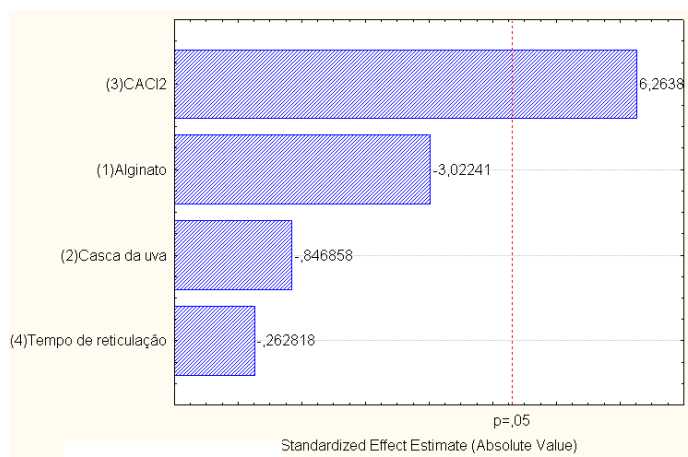

(B)

Figura 2. Diagrama de Pareto para o grau de intumescimento (A) e grau de erosão (B) do encapsulado seco na estufa de cada variável do planejamento fatorial facionado $2^{4-1 .}$

Os melhores resultados encontrados foram no experimento 1 da tabela 2 e no 2 da tabela 3 , no qual foi obtido o menor grau de erosão, aquelas em torno de $20 \%$. Portanto quanto menor o grau de erosão, maior será o tempo para desintegrar o encapsulado dentro do espumante. Vale ressaltar que visualmente não foram observados fragmentos dos polímeros nos ensaios de erosão.

O tempo de sedimentação foi outro parâmetro a ser analisado. Dentre as esferas secas na estufa o maior tempo de sedimentação foi encontrado no experimento $1 \mathrm{com} 2,99$ segundos e o menor no experimento 5 com 1,10 segundos. Analisando as esferas secas pelo método de liofilização encontramos sedimentação a partir do sétimo dia nos experimentos 1,2,5,6 e 7, porém os mesmos ainda apresentam material flutuando, não tendo sedimentado a sua totalidade.

\section{REFERENCIAS}

ANJANI, K.; KAILASAPATHY, K.; PHILLIPS, M. Microencapsulation of enzymes for potential application in acceleration of cheese ripening. International Dairy Journal, v.17, p. 7986, 2007.

ANSEL, H. C., POPOVICH, N. G., ALLEN JR., L. V., Farmacotécnica: Formas Farmacêuticas E Sistemas De Liberação De Fármacos. São Paulo: Editorial Premier, p.484 1999.

CAMPOS L. M.A.S .Obtenção de extratos de bagaço de uva Cabernet Sauvignon (Vitis vinifera):Parâmetros de processo e modelagem matemática. Dissertação (Mestrado Engenharia de alimentos), Santa Catarina: p. 141, 2005. 
CATANEO, C.B.; CALIARI V.; GONZAGA L. V.; KUSKOSKI E. M.; FETT, R. Atividade antioxidante e conteúdo fenólico do resíduo agroindustrial da produção de vinho. SEMINARIO: CIÊNCIAS AGRÁRIAS, Londrina, v. 29, n. 1, p. 93-102, jan./mar. 2008.

EFENTAKIS, M., KOUTLIS, A.,VLACHOU, M., Development and evaluation of oral multiple unit and single unit hydrophilic controlled release systems. AAPS PharmSci Tech, 1, E34(2000)

MONTGOMERY, D. C. Design and analysis of experiments. 4th ed. New York: John Wiley \& Sons, 1999. 704p.

NETO, B. B.; SCARMÍNIO, I.; BRUNS, R. E. Planejamento e otimização de experimentos. Campinas: Editora Unicamp, 1995. 299p.

RIZZON, L.A.; MANFROI, V.; MENEGUZZO, J. Elaboração de suco de uva na propriedade vitícola. Bento Gonçalves: Embrapa Uva e Vinho. 22 p., 1998.

SCALBERT, A.; WILLIAMSON, G. Dietary intake and bioavailability of polyphenols. Journal of Nutrition, v.130, n.8 (supl.), 2000.

SGARBIERI, V.C.; PACHECO, M.T.B. Revisão: Alimentos funcionais fisiológicos. Brazilian Journal of Food Technology, v.2, n.1-2, p.7-19, 1999.

YU, C. Y.; YIN, B. C.; ZHANG, W.; CHENG, S. X.; ZHANG, X. Z.; ZHUO, R. X. Composite microparticle drug delivery systems based on chitosan, alginate and pectin with improved $p H$ sensitive drug release property. Colloids and Surfaces B: Biointerfaces, v. 68, pp. 245-249, 2009.

ZACTITI, E. M.; KIECKBUSCH, T. G. Potassium sorbate permeability in biodegradable alginate films: efect of the antimicrobial agent concentration and crosslinking degree. Journal of Food Engineering, v. 77, pp. 462-467, 2006. 